\title{
Análisis contable de una combinación de negocios: la adquisición de Carrefour Colombia por CENCOSUD Chile
}

\author{
Carlos Orlando Rico Bonilla ${ }^{1}$
}

Recibido: 10 de julio de 2014

Aprobado: 17 de octubre de 2014

Rico, C. (2014). Análisis contable de una combinación de negocios: la adquisición de Carrefour Colombia por CENCOSUD Chile. Activos 23, 69-86

Clasificación JEL: M14

\section{Resumen}

En 2012 el grupo empresarial chileno del sector minorista CENCOSUD adquirió por cerca de 2620 millones de dólares la filial en Colombia de la marca francesa Carrefour. El objetivo de este artículo es presentar un breve análisis conceptual de este proceso de combinación de negocios, a la luz de la Norma Internacional de Información Financiera IFRS 3, emitida por el IASB en marzo de 2004, que aplica para este tipo de transacciones.

\section{Palabras clave}

Negocios, CENCOSUD, Carrefour, IFRS 3.

1 Contador público Universidad Nacional de Colombia. Docente de la Facultad de Ciencias Económicas de la Universidad Santo Tomas, consultor en temas relacionados con Estándares Internacionales de Contabilidad. Correo electrónico: coricob@unal. edu.co 
Rico, C. (2014). Accounting analysis of a business combination: The acquisition of Carrefour Colombia by CENCOSUD Chile. Activos 23, 69-86

\begin{abstract}
In 2012 the Chilean business group of the retails sector CENCOSUD acquired for about 2620 million dollars the Colombian subsidiary of the French brand Carrefour. The purpose of this paper is to present a brief conceptual analysis of this business combination process, under the International Financial Reporting Standard IFRS 3, issued by the IASB on March 2004, which applies to such transactions.
\end{abstract}

Keywords

Business, CENCOSUD, Carrefour, IFRS 3.

Rico, C. (2014). Analyse comptable d'un regroupement d'entreprise: l'acquisition de Carrefour Colombie para CENCOSUD Chili. Activos 23, 69-86

\title{
Résumé
}

En 2012, le consortium chilien du secteur minoritaire CENCOSUD a acheté pour près de 2620 millions de dollars la filiale colombienne de la marque française Carrefour. L'objectif de cet article est de présenter une brève analyse conceptuelle de ce processus de regroupement $d$ ' entreprise $d$ ' après la Norme Internationale d'Information Financière IFRS, émise par le IASB en mars 2004, et qui applique pour ce genre de transactions.

\section{Mots clés}

LAffaires, CENCOSUD, Carrefour, IFRS 3. 


\section{Introducción}

En marzo de 2004, el IASB emitió la IFRS 3 Combinación de Negocios como parte del acuerdo de convergencia con el regulador estadounidense, el Financial Accounting Standars Board - $\mathrm{FASB}^{2}$ - . La idea era lograr un mayor entendimiento en cuanto al tratamiento contable más indicado para reflejar adecuadamente las fusiones corporativas y las compras de empresas, líneas de actividad o segmentos.

Este es un tema muy importante en el universo de los mercados de valores globales, ya que se trata de definir los parámetros de reconocimiento, valoración y divulgación de información financiera de transacciones económicas que, por lo general, no corresponden a cuantías menores. Por el contrario, son procesos con algún grado de complejidad y costo que escenifican la misma naturaleza e historia de la economía moderna, sustentada en el precepto clásico de la competencia empresarial. Su regulación contable ha tenido varios ires y venires a lo largo de los últimos sesenta años (Li, 2007).

Por esto no sorprende que de entrada la IFRS 3, en sus términos más básicos, presente ideas muy interesantes: una combinación de negocios, por ejemplo, se entiende como la transacción por medio del cual una empresa "adquiriente obtiene el control de uno o más negocios" (IASB, 2013:175).

En esta definición encontramos dos elementos significativos que permiten una interpretación clara del sentido de la norma. El primero es el principio del control, que es la fuente conceptual que sustenta el objetivo del estándar; y segundo, el método de adquisición ('purchase method'), como la guía operativa para reconocer los elementos de los estados financieros asociados a la transacción, la cual es caracterizada en todo caso como una compra.

2 El estándar convergente es el SFAS No. 141 del Business Combination, cuya versión más actualizada es de 2007. 
El principio de control ha sido recientemente examinado a la luz de los proyectos de norma que derivaron en la publicación de las IFRS 10, 11 y 12, presentándose la reafirmación de conceptos clave como el de influencia significativa y acuerdo conjunto. Al mismo tiempo, se ha enfatizado en el direccionamiento o poder sobre la toma de decisiones de las actividades financieras relevantes como la base del criterio de consolidación de los estados financieros de un grupo económico.

El método de adquisición se estableció como la única opción de política contable, en detrimento del procedimiento de la unión de intereses (pooling method) que permanecía vigente aún en la derogada IAS 22. En realidad, se había cuestionado desde hacía bastante tiempo su aplicación, en tanto que consideraba la combinación como un proceso entre iguales, en el que no se producía reconocimiento de la plusvalía. Se tenían dos procedimientos contables con resultados diferentes para hechos económicos muy similares (Aboody, Kasznik y Williams, 2000; Hopkins, Houston y Peters, 2000).

Además, diferentes investigaciones empíricas realizadas en varios países concluyeron que era probable que las empresas endeudadas que entraran en una combinación tuvieran un incentivo mayor para aplicar el método de la unión de intereses, a fin de aliviar la presentación de resultados y las cargas fiscales. En general, con esta política se mostraba información más conveniente para la empresa adquiriente, que para el fondo económico de la transacción (Giner y Pardo, 2004).

Así, el objetivo del presente documento es continuar con esta argumentación al profundizar en los conceptos del IFRS 3, a la luz del análisis de un caso concreto de combinación de negocios: la compra, por parte del grupo empresarial chileno de la industria minorista CENCOSUD, de la subsidiaria en Colombia del grupo francés Carrefour, producida en el segundo semestre de 2012.

Para lograr este fin, el texto está dividido en cuatro partes: en la primera se describen las compañías partícipes de la operación; en la segunda, la transacción en sí misma, la fecha de transacción y la valoración realizada de 
los activos y pasivos identificables; en la tercera se comenta el tratamiento contable de la plusvalía generada; $y$, finalmente, se formulan las conclusiones.

\section{Identificación del negocio y la adquiriente}

La IFRS 3 indica que para comenzar a evaluar una transacción que potencialmente se debe tratar como una combinación, es necesario identificar que, en efecto, el conjunto de activos y pasivos adquiridos corresponde a lo que se define como un negocio. Es decir "insumos y procesos aplicados a estos insumos que tienen la capacidad de crear productos” (IASB, 2013: 176).

También es necesario reconocer la parte adquiriente, es decir, la compañía que obtiene el control en el proceso de combinación. Adicional a los principios establecidos en la IFRS 10 sobre el particular, la guía de aplicación de la IFRS 3 establece otros parámetros, ya que no siempre es fácil identificar el rol de cada agente en una transacción de esta naturaleza.

Se trata de analizar cuál de las entidades es la que hace la transferencia de activos o incurre en un pasivo para soportar la transacción. Si se efectúa un intercambio de participaciones de patrimonio, es necesario identificar qué sociedad es la emisora de dichos instrumentos, y, salvo los temas de adquisiciones inversas, esta será la adquiriente. Finalmente, hay que considerar el tamaño relativo, ya que se ha demostrado que las mayores adquisiciones en este aspecto son las que se identifican con la parte que inicia la combinación.

En el caso analizado, no se presenta mayor dificultad en este paso. Hay claramente una combinación de negocios. La mezcla de insumos, procesos y productos finales se ajusta completamente a las actividades de ventas al detal que desarrollaba la controlada en Colombia por el grupo Carrefour, más puntualmente por la empresa registrada como Grandes Superficies de Colombia S. A. ${ }^{3}$

3 La participación de la matriz en la subsidiaria correspondía al 100\% (Carrefour, 2012a, p. 104). 
Esta entidad había llegado a tener un considerable crecimiento en el país. Por ejemplo, durante la primera década de este siglo logró una importante expansión de sus más importantes puntos de servicio y ventas, los hipermercados, que llegaron a ser 75. En la figura 1 se observa la evolución de su establecimiento.

Figura 1. Número de hipermercados Carrefour Colombia (2001-2011)

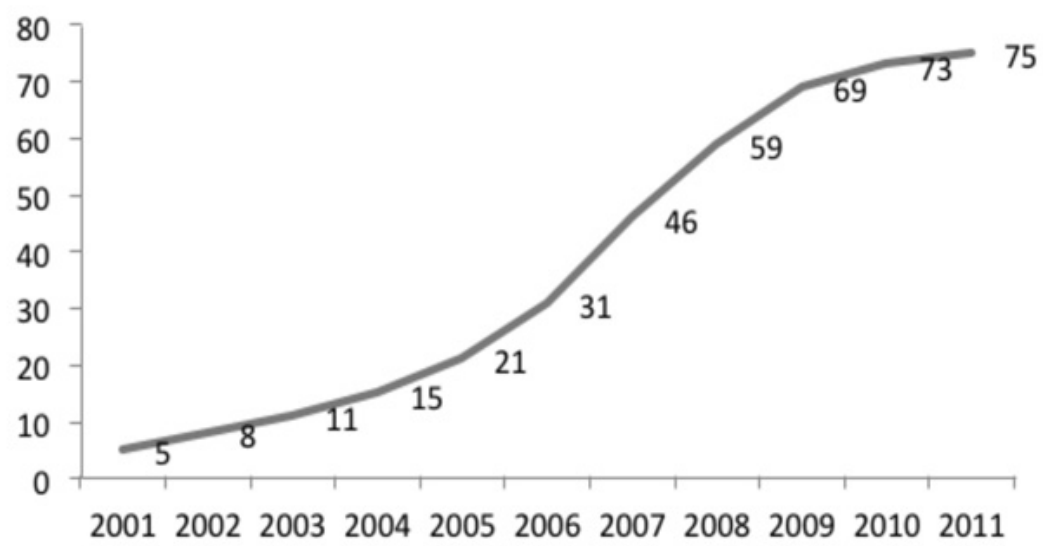

Fuente: elaboración propia a partir de Carrefour (2011, p. 182).

Si bien en Colombia el negocio no marchaba con números negativos -en 2010, las ventas netas alcanzaron la suma de 1544 millones de euros-, en Europa - el entorno originario de la empresa- la crisis financiera y luego las turbulencias económicas de las deudas soberanas sí afectaron el desempeño de la casa matriz francesa y crearon la necesidad de obtener nuevos recursos de financiación a partir de la salida de negocios en otras regiones. Según fuentes oficiales, también fueron vendidas las subsidiarias en Grecia, Singapur, Indonesia y Malasia (El Tiempo, 21 de octubre de 2012; Carrefour, 2012). 
La tabla 1 muestra la reclasificación que realizó en sus estados financieros del cierre de 2012 el grupo Carrefour, al trasladar las mencionadas líneas de negocio a un rubro de operaciones discontinuas, acorde con lo establecido por la IFRS 5, que detalla los principios relativos a activos mantenidos para la venta. Al ubicarse en este rubro, ya no se consolida esta información, pues el control se ha perdido completamente.

Por otra parte, es más que identificable la compañía que actúa como adquiriente; se trata de uno de los holdings más importantes del sector minorista en Chile, y más extensamente en Latinoamérica: el grupo CENCOSUD.

Esta sociedad anónima abierta cuenta con negocios en Argentina, Brasil, Colombia, Perú y Chile, los cuales, según datos estadísticos a 31 de diciembre de 2013, le permiten alcanzar ingresos ordinarios por 19712 millones de dólares (CENCOSUD, 2013a).

La compañía está registrada en las más importantes bolsas de valores de Chile y en la New York Stock Exchange (NYSE) comercializa ADRs (American Depositary Receipt) ${ }^{4}$. Cuenta con cerca de 148.878 trabajadores vinculados. Además del negocio de las ventas al detal, desarrolla operaciones de corretaje de seguros, servicios de fidelización y centros de entretención familiar.

La tabla 2 muestra la distribución patrimonial de las 2'828. 723.963 acciones que componen la estructura del capital. Con un $60 \%$ de la propiedad, el control de la empresa lo tiene la familia Paulmann (CENCOSUD, 2014).

4 Un ADR se define como "un título que se negocia en el mercado de valores de Estados Unidos y representa un número específico de acciones de una compañia establecida por fuera de este país". (Portafolio, 2008). 
Tabla 1. Nota de los estados financieros del cierre 2012 del grupo Carrefour.

\section{RESTATEMENT OF COMPARATIVE INFORMATION}

In accordance with IFRS 5, income and expenses related to discontinued operations (Greece, Colombia, Malasya, Singapore and Indonesia) have been reclassified to "Net income from discontinued operations". In the 2012 and 2011 income statements, and their cash flows have been reclassified to the lines "Impact of discontinued operations" in the 2012 and 2011 statements of cash flows.

\begin{tabular}{c}
\hline DETAILS OF "NET INCOME FROM DISCONTINUED OPERATIONS", \\
"ASSETS HELD FOR SALE" AND “LILIABILITIES RELATED TO ASSETS \\
HELD FOR SALE"
\end{tabular}

The main income statement indicators of the entities reclassified in accordance with IFRS 5 are as follows:

\begin{tabular}{l|c}
\hline (in €millions) & 2012 \\
\hline Net sales & 4,019 \\
\hline Gross margin from recurring operations & 815 \\
\hline Recurring operating loss & $(18)$ \\
\hline Operating loss & $(27)$ \\
\hline Loss before taxes & $(75)$ \\
\hline Income taxes & $(20)$ \\
\hline Net loss & $(95)$ \\
\hline
\end{tabular}

Nota: La tabla contiene la reclasificación de la subsidiaria en Colombia como una operación discontinua.

Fuente: Carrefour (2012, p. 174). 
Tabla 2. Distribución de las acciones que componen el grupo CENCOSUD

\begin{tabular}{c|l|c|c}
\hline \multicolumn{2}{c|}{ Mayores accionistas hasta el 30-06-2014 } & $\begin{array}{c}\text { Número de } \\
\text { Acciones }\end{array}$ & $\begin{array}{c}\text { Participación } \\
\text { porcentual }\end{array}$ \\
\hline 1 & Inversiones Quinchamali Limitada & 573754802 & $20,283 \%$ \\
\hline 2 & Inversiones Latadia Limitada & 550823211 & $19,473 \%$ \\
\hline 3 & Inversiones Tano Limitada & 457879800 & $16,187 \%$ \\
\hline 4 & Banco Santander - JP Morgan & 179115431 & $6,332 \%$ \\
\hline 5 & Banco de Chile por cuenta de terceros & 129607380 & $4,582 \%$ \\
\hline 6 & Banco Itau por cuenta de inversionistas & 105158320 & $3,718 \%$ \\
\hline 7 & Horst Paulmann Kemna & 70336573 & $2,487 \%$ \\
\hline 8 & Fondo de Pensiones Provida C & 56826301 & $2,009 \%$ \\
\hline 10 & Fondo de Pensiones Habitat C & 52369041 & $1,851 \%$ \\
\hline 11 & Fondo de Pensiones Capital C & 41740724 & $1,476 \%$ \\
\hline 12 & Fondo de Pensiones Provida B & 36054975 & $1,275 \%$ \\
\hline 13 & Otros accionistas & 536618587 & $18,970 \%$ \\
\hline & Total & 2828723963 & $100,00 \%$ \\
\hline
\end{tabular}

Fuente: CENCOSUD (2014, p. 11).

\section{Descripción de la transacción, fecha de adquisición y valoración de activos y pasivos}

La IFRS 3 señala que hay que establecer con precisión la fecha de la transacción y la sustancia de aquella. En este sentido, como se ha mencionado, la combinación de negocios fue una adquisición por 2620 millones de dólares. Fue la segunda negociación empresarial más importante de 
2012 en la economía colombiana, luego de la capitalización de Colombia Telecomunicaciones S. A. ESP, que fue por 4128 millones de dólares (Portafolio, 24 de diciembre de 2012).

La fecha de la adquisición fue el 30 de noviembre de 2012, cuando por medio de su subsidiaria, Easy Colombia S. A., la adquiriente compró una participación del $100 \%$, a través de la firma de un contrato de compra de acciones con la filial de Carrefour en Holanda. Para concretar este procedimiento se incurrieron gastos de transacción y servicios legales por cerca de 44 millones de dólares (CENCOSUD, 2013b).

Con esta compra, CENCOSUD pasó a tener en Colombia 72 hipermercados, 16 tiendas y 4 locales en las ciudades más importantes del país, convirtiéndose en el segundo mayor operador de ventas al detal. Además, logró establecer importantes sinergias de ahorros de costos con sus pares de Chile y Perú, aunque en realidad la inversión no se proyectó con perspectivas importantes de crecimiento, a pesar de los buenos resultados que ha experimentado la economía local desde hace algunos años (CENCOSUD, 2013b).

Posteriormente, el estándar IFRS 3 señala que de acuerdo con el método de adquisición debe reconocerse la transacción, tomando como referencia la fecha de ocurrencia de la misma, los activos y pasivos adquiridos, que sean plenamente identificables. Para que estos recursos y obligaciones se puedan incluir en los estados financieros de la adquiriente, es necesario que cumplan con las definiciones de estos elementos, indicadas en el marco conceptual para la presentación de reportes financieros, $\mathrm{y}$, además, que se clasifiquen o designen según sea la aplicación posterior de otras IFRS.

Luego del reconocimiento de los activos y pasivos, se recurre al principio de medición, el cual indica que estos elementos deben asumirse a sus valores razonables en la fecha de adquisición. En este proceso se deben tener en cuenta diferentes criterios que deben ser aplicados a partidas como pasivos contingentes, impuesto a las ganancias, beneficios a empleados, activos de 
indemnización, derechos readquiridos, pagos basados en acciones y activos mantenidos para la venta.

La introducción de la medición a valor razonable a los activos y pasivos que están en el proceso de combinación de negocios no es un tema sencillo, especialmente para elementos que no tienen referencia de su valor en mercados de valores abiertos ni para los que tendrán que utilizar modelos de flujos descontados de caja sustentados en datos no estandarizados.

De hecho, la medición a valor razonable tiene un considerable impacto financiero, si se parte de que en muchas jurisdicciones se aplica el costo histórico como base de la medición de fusiones y escisiones empresariales, y las reglamentaciones tienden normalmente a ser conservadoras en cuanto se debe proteger a los inversionistas minoritarios, cuyos derechos se ven afectados directamente por la combinación de negocios (Stenka, Ormrod y Chan, 2008).

CENCOSUD revela en su reporte financiero consolidado una nota con el resultado final a 31 de diciembre de 2012, sobre la medición a valor razonable del estado de la situación financiera de la empresa adquirida, y lo compara con los saldos preliminares de la empresa adquirida con fecha de corte lo de enero de 2012. De esta manera, es también posible visualizar las partidas que sufren ajustes: cuentas por cobrar y pagar, activos y pasivos por impuestos diferidos, propiedad de planta y equipo, entre otras provisiones. Las tablas 3 y 4 expresan estos datos en miles de pesos chilenos ${ }^{5}$ - los datos no están acompañados de información narrativa que les explique con más detalle-.

Para diciembre de 2013 el departamento contable de Carrefour tomó los estados financieros de Carrefour Colombia, y procedió a cerrar la medición al valor razonable de los activos y pasivos. Para ese año toman protagonismo otra vez las partidas citadas arriba, y se les adicionan ajustes en otros activos y pasivos no financieros, inventarios y activos intangibles.

5 Un peso chileno equivale a 3,59 pesos colombianos (Banco de la República de Colombia). 
Tabla 3. Activos de Carrefour Colombia ajustados a valor razonable en los estados financieros de Cencosud, 2012

\begin{tabular}{|c|c|c|c|}
\hline & $\begin{array}{c}\text { Ajustes del } \\
\text { periodo } \\
\text { ms }\end{array}$ & $\begin{array}{l}\text { Valor final } \\
\text { ajustado a fair } \\
\text { value ms }\end{array}$ & $\begin{array}{c}\text { Valor } \\
\text { preliminar al } \\
01-12-2012\end{array}$ \\
\hline \multicolumn{4}{|l|}{ Activos corrientes } \\
\hline Efectivo y equivalentes al efectivo & 0 & 7137486 & 7137486 \\
\hline $\begin{array}{l}\text { Otros activos no financieros, } \\
\text { corrientes }\end{array}$ & 0 & 2660494 & 2660494 \\
\hline $\begin{array}{l}\text { Deudores comerciales y otras cuentas } \\
\text { por cobrar corrientes }\end{array}$ & -1320346 & 35728423 & 37048769 \\
\hline $\begin{array}{l}\text { Cuentas por cobrar a entidades } \\
\text { relacionadas, corriente }\end{array}$ & 0 & 74099 & 74099 \\
\hline Inventarios & 0 & 109955210 & 109955210 \\
\hline Activos por impuestos corrientes & 0 & 1811403 & 1811403 \\
\hline Activos corrientes totales & -1320346 & 157367115 & 158687461 \\
\hline \multicolumn{4}{|l|}{ Activos no corrientes } \\
\hline $\begin{array}{l}\text { Otros activos no financieros, no } \\
\text { corrientes }\end{array}$ & 0 & 7280 & 7280 \\
\hline $\begin{array}{l}\text { Activos intangibles distintos de la } \\
\text { plusvalía }\end{array}$ & 0 & 7296677 & 7296677 \\
\hline Plusvalía & -26618046 & 0 & 26618046 \\
\hline Propiedades: planta y equipos & 76960623 & 567379985 & 490419362 \\
\hline Propiedades de inversión & 0 & 23495425 & 23495425 \\
\hline \multirow[t]{2}{*}{ Activos por impuestos diferidos } & 1932472 & 34186339 & 32253867 \\
\hline & 52275049 & 632365706 & 580090657 \\
\hline Total de activos & 50954703 & 789732821 & 738778118 \\
\hline
\end{tabular}

Fuente: CENCOSUD (2012, p. 91). 
Tabla 4. Pasivos y Patrimonio de CARREFOUR Colombia ajustados a valor razonable en los Estados Financieros de CENCOSUD, 2012

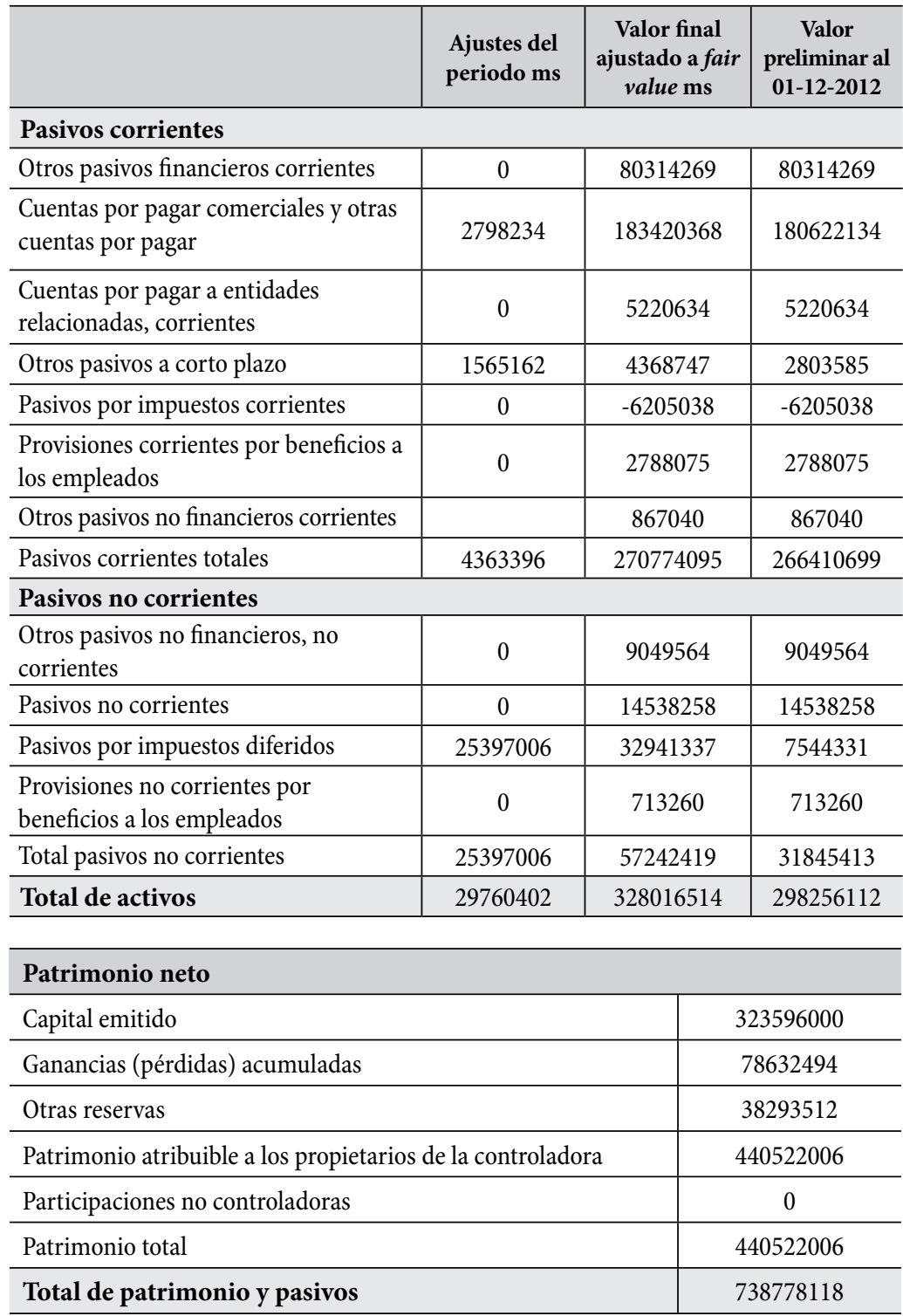

Fuente: CENCOSUD (2012, p. 91). 
En esta ocasión se adiciona información narrativa que da pistas de la medición al valor razonable que se efectúa. Se indica que para los terrenos y edificios se aplicó un valor de mercado (la nota de los estados financieros no señala exactamente cuál).

Por otra parte, para las propiedades, planta y equipo las notas de los estados financieros, afirman que se empleó el enfoque del costo, que define como 'el valor de reposición menos la depreciación'. En el caso de los activos intangibles de clientela y base de datos, se utilizó el método de ingresos excedentes, que consiste en el valor presente de los flujos de caja del activo (CENCOSUD, 2013b). En seguida, se analiza el último paso del tratamiento contable de estas transacciones, fijado por la IFRS 3.

\section{El tratamiento contable de la plusvalía}

La IFRS 3 plantea que luego de realizar el reconocimiento, designación y valoración de los activos y pasivos identificables, así como el registro de los intereses minoritarios, es necesario proceder a reconocer y medir igualmente la plusvalía generada o, por el contrario, una ganancia por compra en términos muy ventajosos, que es asimilable a un ingreso.

En el caso de la combinación de CENCOSUD, no hay lugar para reconocer intereses minoritarios, ya que se adquirió el $100 \%$ de la propiedad y se obtuvo el control completo sobre el negocio. El resultado final de la implementación del método de adquisición en este caso es presentado en los estados financieros del grupo chileno (tabla 5).

Como se puede apreciar, cada año cambia el monto de los activos netos como resultado del ajuste de las partidas iniciales al valor razonable; a este rubro se le resta el precio pagado para obtener la plusvalía, y esta se actualiza, en estas situaciones, sumando la diferencia en cambio, producida en la conversión de pesos colombianos a chilenos. 
Posterior al proceso de reconocimiento y medición inicial, la plusvalía se somete anualmente a pruebas de deterioro, a menos que haya indicios de que es necesario validar con antelación posibles pérdidas por este concepto.

Tabla 5. Plusvalía generada en la combinación de negocios Carrefour ColombiaCENCOSUD, 2012 y 2013, respectivamente, en pesos chilenos

\begin{tabular}{l|c}
\hline Activos netos & $\$ 461.716 .307$ \\
\hline Precio pagado y adeudado en la adquisición & $\$ 1.171 .090 .394$ \\
\hline Plusvalía & $\$ 709.474 .087$ \\
\hline Diferencia acumulada por conversión de moneda & $\$ 29.266 .833$ \\
\hline Plusvalía al 31-12-2012 & $\$ 738.640 .920$ \\
\hline & \\
\hline Activos netos & $\$ 552.934 .377$ \\
\hline Precio pagado y adeudado en la adquisición & $\$ 1.171 .090 .394$ \\
\hline Plusvalía & $\$ 618.156 .017$ \\
\hline Diferencia acumulada por conversión de moneda & $\$ 23.775 .231$ \\
\hline Plusvalía al 31-12-2013 & $\$ 641.931 .248$. \\
\hline
\end{tabular}

Fuente: CENCOSUD (2012, p. 91; 2013, p. 100).

Asimismo, para evaluar con más precisión el valor de este activo intangible, se distribuye porcentualmente entre las unidades generadoras de efectivo que se espera sean beneficiarias de la combinación de negocios, y se procede con el siguiente análisis de cada una de ellas:

[...] se realiza una estimación del valor recuperable de las mismas a través del descuento de los flujos de caja futuros estimados de cada una de ellas. Si el valor recuperable de alguna de las unidades generadoras de efectivo resultase inferior que el de los flujos de caja descontados, se registraría una pérdida con cargo a resultados del ejercicio en que se producen (CENCOSUD, 2012, p. 23). 
Entonces, en 2013 CENCOSUD definió una distribución de la plusvalía del negocio de adquisición en Colombia así: supermercados: 84\%; retail financiero: $10 \%$; y shopping (ventas al por menor a clientes individuales): $6 \%$. En aquel momento no se identificó la necesidad de reconocer deterioros del activo.

\section{Conclusión}

En general en este caso, se aprecia que al implementar IFRS 3 las relevaciones de una empresa adquiriente en una combinación de negocios van ganando relevancia y valor informativo a lo largo del tiempo, en favor de los usuarios de los estados financieros.

No obstante, se aprecian dificultades inherentes al intento de reflejar adecuadamente el proceso, en especial en los aspectos relacionados con la medición del valor razonable de los activos y pasivos que no se transan en mercados abiertos, sino que se terminan midiendo por valor presente o costo.

Otros aspectos no menos completos imprimen cierto grado de dificultad a los demás pasos del método de adquisición definido en la IFRS 3, como son la fecha de la adquisición, las excepciones y, en algunos casos, la definición misma de la entidad adquiriente. Este último aún parece sugerir una visión diferente de la combinación de negocios definida en el procedimiento de registro de la unión de intereses, la cual, a pesar de ser descartada en el marco de la convergencia IASB-FASB, aún suscita algo de controversia.

En el caso analizado, que sin lugar a dudas constituyó una transacción muy importante, no solo para las empresas relacionadas, sino también para la economía doméstica colombiana, se observa que la compra de Carrefour Colombia por parte de CENCOSUD de Chile se ajusta a los pasos del método antes señalado en la normatividad internacional, y su reporte ha ido mejorando con el paso del tiempo. 
Es la expectativa de la profesión contable local, y más allá de la comunidad de negocios, que el proceso de adopción de estos principios y guías de reporte financiero internacional contribuyan al mejoramiento de la calidad de la información, la eficiencia y la transparencia de estos procesos de compra y venta de empresas en nuestro país.

\section{Referencias bibliográficas}

Aboody, D., Kasznik, R., \& Williams, M. (2000). Purchase versus pooling in stock-for-stock acquisitions: Why do firms care? Journal of Accounting and Economics, 29, 261-286.

Carrefour. (2011). Financial Reporting 2011. Consolidated Financial Statement. Ciudad: París, Carrefour.

Carrefour. (2012). Registration Document. Annual Financial Report 2012. Ciudad: París Carrefour.

CENCOSUD. (2012). CENCOSUD S. A. y subsidiarias. Estados financieros consolidados al 31 de diciembre de 2012. Ciudad: CENCOSUD.

CENCOSUD. (2013a). Formulario 20-F. Informe anual en virtud de la sección 13 o 15d de la ley de valores de 1934. Ciudad: CENCOSUD.

CENCOSUD. (2013b). CENCOSUD S. A. y subsidiarias. Estados financieros consolidados al 31 de diciembre de 2013. Ciudad: CENCOSUD.

CENCOSUD. (2014). CENCOSUD S. A. y subsidiarias. Estados financieros intermedios consolidados al 30 de junio de 2014 (no auditado). Ciudad: CENCOSUD.

El Tiempo. (21 de octubre de 2012). Habla el hombre que compró Carrefour Colombia. El Tiempo, p. 15

Financial Accounting Standards Board (FASB). (2007). SFAS No. 141 Business Combinations. Recuperado de www.fasb.org

Giner, B., \& Pardo, F. (2004). La elección del método contable en las fusiones empresariales: Análisis empírico desde una perspectiva contractual. Revista Española de Financiación y Contabilidad, 33(122), 669-703.

Hopkins, P., Houston, R., \& Peters, M. (2000). Purchase, pooling and equity analysts' valuation judgments. The Accounting Review, 75(3), 257-281. 
International Accounting Standards Board (IASB). (2013). Normas internacionales de información financiera - IFRS 3. Recuperado de www.ctcp.gov.co

Li, K. (2007). Trends in accounting for business combinations: A sixty-year review of the jurisdictions. Recuperado de www.ssrn.com

Portafolio. (24 de diciembre de 2012). Conozca las 10 movidas empresariales del 2012 en Colombia. Portafolio, p. 12

Ravina, E. \& Sapienza, P. (2010). What do independent directors know? Evidence from their trading. Review of Financial Studies, 23, 962-1003.

Rico, C. (2004). Globalización, audit expectation gap y rotación obligatoria de auditores. Revista Internacional Legis de Contabilidad y Auditoría, 24, 8-47.

Rozanov, K. (2008). Corporate governance and insider trading. Working Paper, Londres: Business School of finance.

Stenka, R., Ormrod, P., \& Chan, A. (2008). Accounting for business combinations: The consequences of IFRS adoption for UK listed companies. Recuperado de www.ssrn.com 